\title{
Application of Z-Numbers Based Approach to Project Risks
} Assessment

\author{
A.M.Nuriyev \\ Azerbaijan State Oil and Industry University, \\ Azadlyg ave., 20, AZ1010, Baku, Azerbaijan
}

\section{Abstract}

At every phase of the project management process various risks originate owing to occurrence of uncertain events. In this research, we are analyzing potentialities of the Z-numbers in improving the quality of risk assessment. Risk assessment uses probability theory, theory of possibilities, fuzzy approach, Z-number based approach etc. Combined risk measure based on probability and consequence is calculated by applying the disjoint events probability formula or as a product of events. Reliability of relevant information unaccounted in this approach and this circumstance limits the descriptive power of the approach. Suggested by L. Zade a bi-component Znumber $Z=(A, B)$ represents in a unified way a restriction on the values of the uncertain variable (A) and its certainty (B) and allows to take into account the reliability of information. Prediction identical to (High, Very Sure) can be formalized as a Z- evaluation "X is $\mathbf{Z}(\mathbf{A}, \mathbf{B})$ ", where $\mathrm{X}$ is random variable of Risk Likelihood, $\mathbf{A}$ and $\mathbf{B}$ are fuzzy sets, describing soft constraints on a risk likelihood and a partial reliability, respectively. Usually, A and B are sensebased and in effect are imprecise.Z-number describes a probability of threat as: $\quad$ Likelihood $\mathbf{= Z 1}$ (High,Very Sure), where $\mathbf{A}$ is expressed by linguistic terms High, Medium, Low, and B is expressed by terms Very Sure, Sure and so on. Similarly, Consequence measure is described as Consequence measure = Z2 (Low, Sure). Risk levels (Z12) is calculated as the product of the likelihood (Z1) and consequence measures (Z2).Effectiveness of the approach illustrated by examples. A general and computationally effective approach suggested to computation with Z-numbers allows using Zinformation for the solving decision-making problem which can be utilized for risk factors estimation.. ${ }^{1}$ Application of the Z-number based approach for a

\footnotetext{
1 Project management is the multiphase process and at every phase of this process, various risks originate owing to the occurrence of uncertain events. These risks should be identified, assessed and controlled. In the paper, various assessment approaches analyzed and potentialities of the Z-numbers
} 
project risks assessment increases adequacy of the risks representation due to better approximation of the combined effects

Keywords: project management, risk assessment, Z-numbers, risk likelihood, risk consequence, risk level

\section{Introduction}

The project management is becoming an increasingly important tool almost in all areas of human activity. As noted in Gartner and other research agencies reports, about $25 \%$ of world gross product is spent on various kinds of projects. Professional project management allows to effectively distribute responsibilities and duties between project participants, save up to $30 \%$ of time and up to $20 \%$ of funds, reduce project risks (Ashfaque, 2011).

According to the PMI`s Pulse of Profession report for the year 2015 - Capturing the Value of Project Management, on average, 58\% of the respondents fully understand the value of project management approach. Non-project-oriented companies spend about $\$ 250$ million on projects realization, and project-oriented organizations spend thirteen times less-about 20 million a year. High-performing organizations successfully met original goals, complete and implement $90 \%$ of projects in time. Organizations with well-established formal knowledge transfer process significantly improve project outcomes. More than $60 \%$ of organizations carry out regular trainings in project management for their employees (Project Management Institute, 2015;Burba, 2015).

In their daily activities, people encounter and resolve work and life-related issues, and in the most cases these activities are based on purposeful or unconscious use of the project management approach, design and implementation of the various projects: career development, study, moving to another city are the classical examples of such projects. Day-to-day activities of any company are very similar to people`s life: a company develops and implement various projects.

At present many sectors adopt project management approach and become projectoriented and this tendency is increasing. Use of the project management approach in their daily activities allows companies to reach their financial and economic objectives.

All projects are temporary by nature and they have start and end dates. There are various definitions of project management. In many cases, well established international standards define a project management as an application of knowledge,

application in the project risks analysis and evaluation studied. Effectiveness of the approach illustrated by examples. Application of the Z-number based approach for a project risks assessment increases the adequacy of the risk representation due to a better approximation of the combined effects. Use of Z-concept allows taking into account the reliability of the information describing risks. 
skills, techniques, and tools to the project jobs to meet project requirements and specifications. According to the sixth edition of the PMBOK (2017), project management is realized via proper use and integration of the 49 processes grouped in 5 groups and it embraces 10 knowledge areas.

Project management technique has been widely used by financial institutions. These institutions develop and implement various projects in their ongoing daily activities and strategic activities oriented to improvement of business processes. As a result, many publications related to the study of the project risks in one way or another turn to the evaluation of the financial risks or the risks of investment projects. As we mentioned earlier, the project management approach has been used not only in economics and finance, but also in the social, legal, political and other areas. As it mentioned in (Songyan Zhang \& Yuxiao Fang, 2016) the study of risk management in engineering projects primarily based on financial risk management knowledge.

\section{Project risks assessment}

Risks are uncertain events or conditions that directly influence objectives of the project-content, duration, cost, and quality and due to this relationship, risk management is an inseparable part of the project management.

Classification of the project risks can be done in different ways. As a base for the classification can be used frequency of occurrence and the impact scope. A list of the most common risks includes scope, schedule, cost, resources, technology, external hazards, governance, performance, legal issues, etc. (Szymański,2017;Tsiara\&Siakas,2016).Subject area-related specific risks significantly enlarge this list.

The risk management process is a multi-stage, multi-layer and complex procedure. Key stages in the risk management process are risk identification and analysis, response methods development, monitoring and risk management. Risks identification and analysis are one of the important and time-consuming stages of the project management process, they laid down the foundation for the all other stages of the project management and, at the same time, they are the least formalized stages in project management-related decision-making processes. A large amount of research publications analyses project risks as a kernel task of project management processes (Henrique Rodrigues-da-Silva\& Crispim,2014).

At the risk analysis stage researcher should clearly identify inherent to the subject area risks, reveal cause-effect relationships, evaluate level, frequency and consequences of the risks and prioritize risks from the standpoint of the project management decisions. At this stage, various models of the qualitative description of the risks and sub-risks can be used. 


\section{Analysis of the}

(Marle\&Gidel,2012;Rostami,2016;Yesuf,2017;Ungureanu,Braicu\&Ungureanu, 2015;Renault,Agumba \&Ansary,2016) and other research publications shows that there are well-established identification procedures.

Despite availability of the various metrics for the evaluation and description of the risks, quantitative risk analysis is a complicated problem.

In (Aven,2016) the risk definition and metrics from the (SRA Glossary society for risk analysis,2015) are summarized as follows: the combination of probability and severity of consequences; the triplet of the scenario, scenario probability and consequence; the triplet of some specified consequences, measure of consequence uncertainty and background knowledge, supporting consequence and its uncertainty; expected negative consequences computed by expected number of fatalities in a specific period; the product of the hazard occurring probability, the probability of the exposition to the hazard, and the expected damage in case of exposition; expected disutility; a possibility distribution for the damage. It is indicated that suitability of these metrics and descriptions depends on the situation.

Risk assessmentand formalization requires analysis of the uncertainties inherent to the real-world processes and events. This is a hot topic that has been intensively discussed in research publications from the early stages of the risk assessment until today (Aven,2016).

Even though the terms risk and uncertainty have been widely used interchangeable in most part of the surveyed articles, we should take into consideration that these terms have distinct meanings in decision-making models and processes. Term risk is more applicable when information for the evaluation of the likelihood of an event is available, uncertainty more suitable to the case of lack of knowledge (Carvalho,2017).

Unforeseen project risks are mainly caused by uncertainties and these uncertainties can decrease revenue, increase cost, incur losses and so on. In the analysis of longterm projects, it is necessary to take into consideration expected future values of the multiple factors, influencing project baseline, and due to this circumstance getting reliable and accurate forecasts become an insurmountable task. In the forecasting, it is necessary to differentiate and to take into consideration the uncertainties causing risks for the given measures of effectiveness, as a result, arises an issue of the formalization of forecasted uncertain characteristics of the project and their appropriate estimation. So, the presence of the various types of uncertainties necessitates adaptation of the project effectiveness measures. This adaptation can be done by use of the appropriate mathematical tools, allowing to formalize and process various types of the uncertainties.

Risk assessment uses various mathematical tools: probability theory, theory of possibilities, fuzzy approach, etc. Historically, probabilities were the first tools used for the formalization of the uncertainties in decision making. Probability is the most 
common tool, but other tools also exist, including imprecise (interval) probability and representations based on the theories of possibility and evidence, as well as qualitative approaches (Aven,2016;Zhang,Li\&Zhang,2016;Ghasemi,Hossein Mahmoudi Sari,Yousefi,Falsafi\&Tamosaitiene,2018).

Quantitative possibility theory allows reconciliation of the probability and fuzzy approach. A current major concern in risk analysis studies is uncertainty propagation under poor data and without independence assumptions. Methods for joint propagation of possibilistic and probabilistic information and probabilistic models with fuzzy interval parameters have been developed (Dubois\&Prade,2015).

In (Zlateva,2015) the fuzzy logic method for assessment of risk management capability is proposed. The fuzzy logic method is developed as a hierarchical system with several inputs and one output. The proposed method for the assessment of risk management capability is envisaged to be implemented as a part of the information system for integrated risk management of natural disasters.

Zadeh (2011 p.1) noted that "In the real world, uncertainty is a pervasive phenomenon. Much of the information on which decisions are based is uncertain. Humans have a remarkable capability to make rational decisions based on information which is uncertain, imprecise and/or incomplete. Formalization of this capability, at least to some degree, is a challenge that is hard to meet. It is this challenge that motivates the concepts and ideas outlined in this note". In this note, the concept of a Z-number is introduced and methods of computation with Z-numbers are outlined

Suggested by Zadeh a bi-component Z-number $\mathrm{Z}=(\mathrm{A}, \mathrm{B})$ represents in a unified way a restriction on the values of the uncertain variable $(A)$ and its certainty $(B)$.

Since its introduction, the concept of Z-numbers has been successfully applied as a new direction in the analysis of uncertain and complex systems in various areas of science and technology.

The main critical problems that naturally arises in processing Z-information are computation and reasoning with Z-information (Aliev, Alizadeh\&Huseynov, 2015).

\section{Fuzzy approach to risk assessment}

Until recently the applications of Z-numbers based approaches were limited by the lack of efficient technique for computations with Z-numbers.As it was noted by L.Zadeh (2011 p.5) «Problems involving computation with Z-numbers are easy to state but far from easy to solve».

Kang et al.(2012) suggest an approach based on converting a Z-number to a fuzzy number on the base of an expectation of a fuzzy set. However, converting Z-numbers to fuzzy numbers leads to loss of original information reducing the benefit of using original Z-number-based information (Aliev,Huseynov,Aliev\&Alizadeh,2015). 
The work of Zadeh (2012) discusses different methods, applications, and systems based on the Z-number concept. Aliev and colleagues suggested a general and computationally effective approach to computation with Z-numbers. The approach is applied to the computation of arithmetic and algebraic operations, t-norms and snorms, and construction of typical functions (Aliev,Huseynov\&Zeinalova,2016; Aliev et al., 2015; Aliev,Huseynov\&Aliyev,2017;Aliev, Huseynov\&Alieva,2016)

In (Cooper,Grey,Raymond\&Walker,2005) combined risk measures based on risk likelihood and risk consequence are described. The method applies disjoint events probability or product formula. In this approach, the descriptive likelihood assessments are converted to numerical measures and the numerical measures are averaged, to get a risk likelihood measure P. A similar process is used for the consequence assessments, to get an average consequence measure $\mathrm{C}$. A combined risk measure $\mathrm{RF}$ is then calculated for each risk. $\mathbf{P}=$ risk likelihood measure, on a scale 0 to 1 =average of likelihood factors; $\mathbf{C}=$ consequence measure, on a scale 0 to $1=$ average of consequence factors:

\section{$\mathbf{R F}=$ risk factor $=\mathrm{P}+\mathrm{C}-\left(\mathrm{P}^{*} \mathrm{C}\right)$}

The risk factor RF, from 0 (low) to 1 (high), reflects the likelihood of a risk arising and the severity of its impact.

In some circumstances, risk factors may be calculated as the product of the likelihood and consequence measures:

\section{$\mathbf{R F}=\mathbf{P}^{*} \mathbf{C}$}

The 'product' formula has one significant disadvantage in comparison with the earlier form - items with high consequences but low probabilities may be allocated low-risk factors, and hence they may not be flagged as important. This can be a problem in practice, as there is a chance that significant risks may not be noticed. The earlier version identifies items with high likelihoods or high consequences or both, so the chance of high consequence but low likelihood items being ignored is reduced greatly.

Product of the threat likelihood and threat impact is proposed for risks level calculating in (Stoneburner, Goguen\& Feringa,2002; NIST,2012) where it is proposed to product Threat Likelihood (High, Medium, and Low - for example) and Impact Level (High, Medium, and Low):

\section{Risk level $=$ Threat Likelihood*Impact Level}

The determination of these risk levels or ratings may be subjective. The rationale for this justification can be explained in terms of the probability assigned for each threat likelihood level and a value assigned for each impact level. For example, the probability assigned for each threat likelihood level is 1.0 for High, 0.5 for Medium, 0.1 for Low. The value assigned for each impact level is 100 for High, 50 for Medium, and 10 for Low. The quantifiers Very High and Very Low can be used as well. 
Let's consider using Z-numbers to calculate the level of risk or risk factors. In many areas, risk experts deal with the prediction like this one (High, Very Sure). This prediction can be formalized as a Z-number-based evaluation " $\mathrm{X}$ is $\mathrm{Z}(\mathrm{A}, \mathrm{B})$ ", where $\mathrm{X}$ is a random variable of Risk Likelihood, $\mathrm{A}$ is a fuzzy set used to describe soft constraint of risk likelihood and $B$ is a fuzzy number to describe a soft constraint on a partial reliability of $\mathrm{A}$. As Zadeh noted " $\mathrm{X}=\mathrm{Z}(\mathrm{A}, \mathrm{B})$ is Z-valuations. A collection of Zvaluations is referred to as Z-information". It should be noted that much of everyday reasoning and decision-making is based on Z-information.

Probability of threat can be described via Z-numbers as:Likelihood=Z1(High,Very Sure), where a value of the A is expressed by linguistic terms High, Medium, Low, and avalue of the B isexpressedbylinguisticterms Very Sure, Sure and so on. Similarly, Consequence measure can be described as Consequence measure $=\mathbf{Z 2}$ (Low, Sure).

The example of encoded linguistic terms for A component of Z-numbers

\begin{tabular}{|l|l|l|}
\hline Scale & Level & $\begin{array}{l}\text { membership function } \\
\text { value (triangle) }\end{array}$ \\
\hline 1 & Very Low & $1 / 1,1 / 1,0 / 0$ \\
\hline 2 & Low & $0 / 1,1 / 2,0 / 3$ \\
\hline 3 & Medium & $0 / 2,1 / 3,0 / 4$ \\
\hline 4 & High & $0 / 3,1 / 4,0 / 5$ \\
\hline 5 & Very high & $0 / 4,1 / 5,1 / 5$ \\
\hline
\end{tabular}

The example of encoded linguistic terms for B component of Z-numbers

\begin{tabular}{|l|l|l|}
\hline Scale & Level & $\begin{array}{l}\text { membership function } \\
\text { value (triangle) }\end{array}$ \\
\hline 1 & Unlikely & $1 / 0,05,1 / 0,05,0 / 0,25$ \\
\hline 2 & Not very likely & $0 / 0,05,1 / 0,25,0 / 0,5$ \\
\hline 3 & Likely & $0 / 0,25,1 / 0,5,0 / 0,75$ \\
\hline 4 & Very likely & $0 / 0,5,1 / 0,75,0 / 1$ \\
\hline 5 & Extremely likely & $0 / 0,75,1 / 1,1 / 1$ \\
\hline
\end{tabular}

As it is shown in (Stoneburner et al.,2002) risk levels (Z12) can be calculated as the arithmetic product of the Z-numbers (Aliev\&Salimov,2017) likelihood (Z1) and consequence measures (Z2):

\section{$\mathbf{Z 1 2}=\mathbf{Z 1} * \mathbf{Z 2}$}

For example Z-numbers expressing the values of a risk factor - violation of terms of delivery - can be calculated as a product of Z-number - Likelihood (medium, very likely) and Measure of consequences (Low, Sure)

\section{Risk Factor (violation of terms of delivery) = Likelihood (High, Very Sure) * Consequence (Low, Sure)}

In order to evaluate the risk factors (risk levels) and to make a decision, the operation of the ranking of Z-numbers (Aliev,Huseynov O \&Serdaroglu, 2016) is used. 
The analysis of the values of different Z1 and Z2, allows to determine when the risk is greater, for example: Likelihood (High, Very Sure)*Consequence (Low, Very Sure) or Likelihood (Very High, Sure)*Consequence (Medium, Sure).

Arithmetic operations on Z-numbers as well as the ranking of Z-numbers and aggregation of Z-information allow using Analytical Hierarchy Process (AHP) and Multi-Criteria Decision Analysis (MCDA) (Chatterjee\&Kar,2018;Gardashova,2014) for the solving decision-making problem which can be utilized for risk factors estimation.

Also, taking into account successful applications of fuzzy IF-THEN rules for solving a wide spectrum of real-world problems, Zadeh $(2011,2013)$ provides a brief discussion on crucial importance of the development of Z-rules - IF-THEN rules with Z-number valued components, which naturally would have expressive power.

In (Aliev,Pedrycz,Huseynov\&Eyupoglu,2017) a new approach is developed to study approximate reasoning with Z-rules on a basis of linear interpolation. Research team provides an application of the approach to job satisfaction evaluation and to students' educational achievement evaluation problems related to psychological and perceptual issues naturally characterized by imperfect information. The obtained results show the applicability and validity of the proposed approach. In (Abiyev,Uyar,Ilhan,Imanov\&Abiyeva,2018) Z-number-based fuzzy If-Then rules are applied for the determination of the food security risk level in Turkey.

\section{Conclusion}

At every phase of the project management process, various risks originate owing to the occurrence of uncertain events. Uncertainty of the project information is caused by the complexity of the project nature - project is a unique, temporary and progressively elaborating endeavor. Risks directly influence objectives of the projectcontent, duration, cost, and quality and due to these relationships risk management is an inseparable part of the project management. Risks should be identified, assessed and controlled.

Risk assessment tools based on traditional probabilistic or possibility models have a limited capacity of description and processing project-related uncertain information and not in all cases are relevant for the risk assessment. Recent advances in computation with Z-numbers allows to conceptualize and process uncertain information by using perception-based and linguistically expressed fuzzy numbers, describing both restriction on the value of the uncertain variable and reliability of the value.

Risk analysis includes risks that can be evaluated based on the past statistical data and risks that are an inherent part of the given situation without past data available. Evaluation of such risks is based on unconscious experience and intuition. Recently developed and based on arithmetic operations on Z-numbers, the ranking of Z- 
numbers and aggregation of Z-information, as well as Z-rules approach, allows to refrain from excessive assessment simplification and to successfully resolve issues of the multi-risk situations analysis without loss of contained in Z-numbers information. The aim of further research is the solution to the various project risk analysis problems.

\section{References}

[1] Abiyev Rahib H., Kaan Uyar, Umit Ilhan, Elbrus Imanov, and Esmira Abiyeva, "Estimation of Food Security Risk Level Using Z-Number-Based Fuzzy System," Journal of Food Quality, vol. 2018, Article ID 2760907, 9 pages, 2018. https://doi.org/10.1155/2018/2760907.

[2] Aliev R.A., Alizadeh A.V., Huseynov O.H. (2015). The arithmetic of discrete Znumbers. Inform. Sciences 290, 134-155

[3] Aliev R.A., O.H.Huseynov, L.M.Zeinalova,The arithmetic of continuous Znumbers,InformationSciences,Volume 373,2016,Pages 441-460,ISSN 00200255,https://doi.org/10.1016/j.ins.2016.08.078.

[4] Aliev R.A., O.H.Huseynov, R.R.Aliev, A.V.Alizadeh. The Arithmetic of ZNumbers: Theory and Applications. World Scientific, 2015

[5] Aliev R.A., O.H.Huseynov, R.R.Aliyev, A sum of a large number of Znumbers,Procedia Computer Science, Volume 120, 2017, Pages 16-22

[6] Aliev R.A., O.H.Huseynov, K.R.Alieva. (2016). Z-valued T-norm and T-conorm Operators-based aggregation of Partially Reliable Information. Procedia Computer Science. 102 (2016) 12-17

[7] Aliev R. A., W. Pedrycz, O. H. Huseynov and S. Z. Eyupoglu, "Approximate Reasoning on a Basis of Z-Number-Valued If-Then Rules," in IEEE Transactions on Fuzzy Systems, vol. 25, no. 6, pp. 1589-1600, Dec. 2017. doi: 10.1109/TFUZZ.2016.2612303

[8] Aliev, Rafik\&Salimov, V.H. (2017). Software for Z-arithmetic. Procedia Computer Science. 120. 290-295. 10.1016/j.procs.2017.11.241.

[9] Aliev, Rafik \&Huseynov, 0 \&Serdaroglu, R. (2016). Ranking of Z-Numbers and Its Application in Decision Making. International Journal of Information Technology \& Decision Making. 15. 1-17. 10.1142/S0219622016500310.

[10] Ashfaque Ahmed.Software Project Management: A Process-Driven Approach,: Auerbach Publications, 2011, 456 pages

[11] Aven T., Risk assessment and risk management : Review of recent advances on their foundation, European Journal of Operational Research (2016), http://dx.doi.org/10.1016/j.ejor.2015.12.023

[12] Burba, Donovan (2015). Focus on the fundamentals. PM Network, April 2015, Volume 29, number 4. Retrieved from http://www.pmnetworkdigital.com/pmnetwork/april_2015?pg=1\#pg1

[13] Carvalho, Marly. (2017). Risk and uncertainty in projects management: literature review and conceptual framework RECEBIDO. GEPROS. Gestão da Produção, Operações e Sistemas. 12. 93-120. 10.15675/gepros.v12i2.1637. 
[14] Chatterjee, K., \&Kar, S. (2018). A multi-criteria decision making for renewable energy selection using Z-numbers in uncertain environment.Technological and Economic Development of Economy,24(2),739-764. https://doi.org/10.3846/20294913.2016.1261375

[15] Cooper, D., Grey, S., Raymond G. and Walker, P. (2005). Project Risk Management Guidelines, "Managing Risk in Large Projects and Complex Procurements,JohnWiley\&Sons,Ltd ISBN 0-470-02281-7

[16] Henrique Rodrigues-da-Silva, Luiz \& Crispim, José. (2014). The Project Risk Management Process, a Preliminary Study. Procedia Technology. 16. 943-949. 10.1016/j.protcy.2014.10.047.

[17] Gardashova, L. 2014. Application of operational approaches to solving decision making problem using Z-numbers, Applied Mathematics 5: 13231334. https://doi.org/10.4236/am.2014.59125

[18] Ghasemi, Foroogh\&Hossein Mahmoudi Sari, Mohammad \&Yousefi, Vahidreza\&Falsafi, Reza \&Tamosaitiene, Jolanta. (2018). Project Portfolio Risk Identification and Analysis, Considering Project Risk Interactions and Using Bayesian Networks. Sustainability. 10. 1609. 10.3390/su10051609.

[19] A Guide to the Project Management Body of Knowledge, PMBOK Guide, Project Management Institute, 6-th edition, 756p, 2017

[20] Kang, BingYi\& Wei, D \& Li, Y \& Deng, Y. (2012). Decision making using Znumbers under uncertain environment. Journal of Computational Information Systems. 8. 2807-2814.

[21] Marle F., Thierry Gidel. Assisting Project Risk Management Method Selection. International Journal of Project Organisation and Management, 2012. 〈hal-01204820〉

[22] NIST Special Publication 800-30, 2012

[23] Project Management Institute (2015). Pulse of the Profession Capturing the Value of Project Management. Retrieved from https://www.pmi.org//media/pmi/documents/public/pdf/learning/thought-

leadership/pulse/pulse-of-the-profession-2015.pdf

[24] Renault, B.Y., Agumba, J.N \&Ansary, N. 2016. A theoretical review of risk identification: perspective of construction industry.5th Applied Research Conference In Africa (ARCA 2016), At Cape Coast, Ghana, Volume: 773-782 http://hdl.handle.net/10210/214956

[25] Rostami Ali. "Tools and Techniques in Risk Identification: A Research within SMEs in the UK Construction Industry." Universal Journal of Management 4.4 (2016) 203 - 210. doi: 10.13189/ujm.2016.040406.

[26] Songyan Zhang, Yuxiao Fang. Literature Review on Engineering Project Risk Management. Conference Paper.Proceedings of the 2016 5-th International Conference on Sustainable Energy and Environment Engineering (ICSEEE 2016) https://doi.org/10.2991/icseee-16.2016.26

[27] SRA Glossary society for risk analysis:www.sra.com/resources (2015). 
[28] Stoneburner Gary, Alice Goguen, and Alexis Feringa. Risk management guide for information technology systems. Recommendations of the National Institute of Standards and Technology.U.S. DEPARTMENT OF COMMERCE NİST SP 800-30, 2002

[29] Szymański, Paweł. (2017). Risk management in construction projects. Procedia Engineering. 208. 174-182. 10.1016/j.proeng.2017.11.036.

[30] Tsiara, Eleana \& Siakas, Kerstin. (2016). Investigating the Application of Risk Management in

Greek

IT

Companies.https://www.researchgate.net/publication/327602021

[31] Ungureanu Anca\&Braicu Cezar\& Adrian Ungureanu, 2015. Risk Identification in Project Management,International Conference on Economic Sciences and Business Administration, SpiruHaret University, vol. 2(1), pages 259-266, December.

[32] Yesuf, Ahmed. (2017). A Review of Risk Identification Approaches in the Telecommunication Domain. 10.5220/0006197603890396.

[33] Zhang, Zhan \& Li, Kai \& Zhang, Lei. (2016). Research on a Risk Assessment Method considering Risk Association. Mathematical Problems in Engineering. 2016. 1-7. 10.1155/2016/9191360.

[34] Zadeh Lotfi A., A Note on Z-numbers, Information Sciences, Volume 181, Issue 14, 2011, Pages 2923-2932, ISSN 0020-0255, https://doi.org/10.1016/j.ins.2011.02.022.

[35] Zadeh, L. A., 2012. Methods and Systems for Applications forZ-Numbers, United States Patent, Patent No.: US 8,311,973 B1, Date of Patent: Nov. 13, 2012

[36] Zadeh Lotfi A. Z-NUMBERS-A NEW DIRECTION IN THE ANALYSIS OF UNCERTAIN AND COMPLEX SYSTEMS. 7 th IEEE International Conference on Digital Ecosystems and Technologies, July 24, 2013 Menlo Park

[37] Zlateva, Plamena\&Velev, Dimiter\&Raeva, L. (2015). A Fuzzy Logic Method for Assessment of Risk Management Capability. International Journal of Innovation, Management and Technology. 6. 260-266. 10.7763/IJIMT.2015.V6.612. 